\title{
Normocalcemic primary hyperparathyroidism: A newly emerging disease needing therapeutic intervention
}

\author{
Gonzalo Díaz-Soto, ${ }^{1}$ María Teresa Julián, ${ }^{2}$ Manel Puig-Domingo ${ }^{2}$ \\ ${ }^{1}$ Hospital Clínico y Universitario de Valladolid, IEN-Universidad de Valladolid, ${ }^{2}$ Hospital Universitari Germans Trias \\ i Pujol, Badalona (Barcelona), Spain
}

\section{INTRODUCTION}

Normocalcemic primary hyperparathyroidism (NCHPT) is a new entity which possibly represents a "fruste form" of classic clinically symptomatic hyperparathyroidism. Over the last decade it has generated considerable scientific interest. ${ }^{1}$ However, its worldwide official recognition was announced recently as in 2008 when an international workshop on primary hyperparathyroidism took place in order to clarify its nature and relevance. ${ }^{2}$

Its true prevalence, natural history and clinical significance are mostly unknown, although its diagnosis is becoming more common in different clinical settings, from internal medicine outpatient clinics to endocrine or rheumatologists consultations and even at the primary care level. The natural history of this condition remains unknown and not well understood, and very few prospective studies have tried to describe it. ${ }^{3}$ What is more, recent consensus on NCHPT did not specify the follow-up or treatment requirements.

Key words: Asymptomatic, Hyperparathyroidism, Normocalcemic

Address for correspondence:

Gonzalo Díaz Soto, Hospital Clinico Universitario de

Valladolid, Servicio de Endocrinología, Edificio Rondilla,

Calle Rondilla de Santa Teresa s/n. 47005, Valladolid, Spain,

Tel.: +34 983420000 (Ext 21615),

e-mail: diazsotogonzalo@gmail.com

Received 12-05-12, Revised 08-07-12, Accepted 07-10-12
For this reason, it is a challenging situation for both the physician and the patient, as therapeutic recommendations are not as yet established and different patients may be advised to receive a particular active treatment or just follow-up, or even no follow-up. This review presents the current data in relation to this newly emergent condition.

\section{DEFINITION AND BIOCHEMICAL CONSIDERATIONS}

NCHPT is defined as a condition in which persistently normal serum calcium levels are observed in the presence of high levels of parathyroid hormone (PTH). The term NCHPT was first used in the 1960's by Wills et $\mathrm{al}^{4}$ who described a group of patients with different characteristics from those with classic primary hyperparathyroidism (HPT) and in whom clinical symptoms and signs may manifest, while hypercalcemia is absent. No other terms have been used in the definition of this condition, such as subclinical hyperparathyroidism or low grade primary hyperparathyroidism, as has been the case for similar endocrine disturbances (as, for example, subclinical hypothyroidism). The definition of NCHPT includes total albumin-corrected and ionized serum calcium within the normal range and elevated PTH. Specifically, both ionized and serum calcium within the normal levels is required for NCHPT diagnosis. Thus, studies in which ionized calcium levels were not evaluated should not be considered accurate enough for the evaluation of NCHPT with confidence. ${ }^{5}$ 
Under such a biological combination, a rigorous search for causes of secondary hyperparathyroidism syndrome is the first step that the clinician is required to undertake. In particular, vitamin D deficiency, a condition that is very prevalent all over the world, ${ }^{6}$ needs to be ruled out before the diagnosis of NCHPT may be made. This is in fact a major issue, as in most cases it is not known if the reference range of a given PTH assay has been made in a population in which vitamin D deficiency exclusion criteria have been considered. What is more, clinical assays for vitamin $\mathrm{D}$ have been subject to inaccuracy, although results are likely to improve in precision as uniform standards, made available through the National Institute of Standards and Technology, become more widely implemented.

It should be noted here that in NCHPT an inverse relationship between 25 -hydroxivitamin $\mathrm{D}$ and PTH exists. When vitamin $\mathrm{D}$ decreases below the normal range, the parathyroid gland responds with increased synthesis and secretion of PTH, which should subsequently be normalized after replacement treatment. The level of 25-hydroxivitamin D at which this occurs is somewhere between 20 and $30 \mathrm{ng} / \mathrm{mL} .{ }^{7}$ However, this form may represent a masked form of classic hypercalcemic HPT masked by vitamin D deficiency and patients would become hypercalcemic as soon as their vitamin D levels are replaced.

Parathyroid hormone is a heterogeneous molecule and, in the last 25 years, its measurement has been performed with different assays which have been tried out in the course of technological advances in this field. For the past few years our institution has been using a third-generation assay. The sensitivity of second and third-generation PTH assays in detecting elevated PTH values seems to be similar. ${ }^{8}$ By using a second-generation PTH assay it has been recognized how important it is to exclude subjects with low serum 25-hydroxivitamin D levels in establishing a reference range for $\mathrm{PTH} .{ }^{9}$ When this is taken into account, the upper limit of the PTH reference interval for secondgeneration assays decreases from 65 to $46 \mathrm{ng} / \mathrm{liter}$, a $29 \%$ reduction. The same applies for establishing a reference interval for "whole PTH" assays, the upper limit decreasing from 44 to $34 \mathrm{ng} / \mathrm{L}$, a $27 \%$ reduction. Finally, the upper limit of the reference interval also remains lower for third-generation assays than for second-generation ones. It has to be mentioned that in parallel, vitamin D references values have been established over the last few decades, though however there is still a debate going on in relation to what are the lower normal values and what is the importance of seasonal changes of the normality. ${ }^{10}$ The result is that at present no consensus exists as to the normal range of vitamin $\mathrm{D}$ concentrations in the general population. In some studies, values below $20 \mathrm{ng} / \mathrm{mL}$ were taken as the lower limit of vitamin D sufficiency, whereas other studies have reported that PTH levels in normocalcemic individuals continue to decline until levels of 25-hydroxivitamin D above $30 \mathrm{ng} / \mathrm{ml}$ have been achieved. ${ }^{11-13}$ This emphasizes the importance of establishing an international consensus on the reference range for 25-hydroxivitamin $\mathrm{D}$ if we are to improve the accuracy of the reference range of PTH by excluding subjects with vitamin D insufficiency. A very interesting approach has recently been proposed by Harvey et $\mathrm{al}^{14}$ who suggested using a nomogram in which vitamin $\mathrm{D}$ and age are included in order to refine the diagnosis of primary hyperparathyroidism.

In addition to excluding vitamin D deficiency, it is also important to rule out other causes of secondary elevations in PTH, such as urinary calcium leak and reduced creatinine clearance. ${ }^{7}$

\section{EPIDEMIOLOGY}

There are virtually no data regarding the epidemiology of NCHPT. Lundgren et al ${ }^{15}$ studied more than 5,000 postmenopausal women between the ages of 55 and 75 in a Swedish survey. They found that $16 \%$ of individuals had normal serum calcium levels $(<9.9 \mathrm{mg} / \mathrm{dL})$ and elevated PTH. This group included both those with vitamin D deficiency, which was not excluded, and the non-deficient cases, which should be considered as the true cases of NCHPT. It should be noted here that the combined prevalence of both causes of high PTH is indeed surprising, but more data are needed in order to confirm such a surprisingly high prevalence.

\section{CLINICAL PRESENTATION}

Usually, NCHPT is identified in the context of an evaluation of patients with low bone density in specialized metabolic bone units, in whom secondary 
causes for increased PTH concentration have been carefully ruled out. However, some patients with NCHPT are diagnosed because of fragility fracture, nepholitiasis, hypercalciuria, high serum PTH values or an incidental discovery of an enlarged parathyroid on cervical ultrasonography., ${ }^{3,16}$

It is unknown if NCHPT is an indolent disease or if it may have an impact similar, to a certain extent, to that of classic primary hyperparathyroidism as a consequence of chronically elevated levels of circulating PTH. There are few studies available that have evaluated the long-term evolution of this entity and they present inconclusive data regarding the progression and the clinical consequence of this condition ${ }^{1,17-18}$ (Table 1). Moreover, in several studies secondary causes of PTH elevation have not been rigorously excluded. ${ }^{15-19}$ Recently, Silverberg and Bilezikian have demonstrated that patients with NCHPT are at risk of disease progression, further developing features of classical hyperparathyroidism in certain cases, such as low bone mass, fragility fracture and kidney stones. Data also suggest that some of these patients will become hypercalcemic over time. ${ }^{1,18}$

In a major observational study by Lowe et al in which causes of secondary hyperparathyroidism were ruled out, 37 patients who met the criteria of NCHPT were followed up for a mean of 3yrs (range, 1-8yrs). ${ }^{18}$ At the initial evaluation, most of the patients had clinical features of classical PHPT: $57 \%$ had osteoporosis in at least one site, $14 \%$ nepholitiasis and $11 \%$ fragility fracture. Osteoporosis was more common at the lumbar spine and hip than at the distal radius, in contrast to classical PHPT where preponderance of bone density loss is observed more often at the cortical radius. In this cohort, $40 \%$ of the patients showed evidence of progressive hyperparathyroid disease, developing nephrolithiasis, (3\%) marked hypercalciuria $(5 \%)$ or progressive cortical bone loss, $(29 \%)$ and even fractures. (3\%) Moreover, 7 individuals $(19 \%)$ developed hypercalcemia during the follow-up, and it was observed that those with the highest average serum calcium concentrations and those who were somewhat older were more likely to develop hypercalcemia. The authors raise the possibility that these patients' condition may represent the earliest form of the symptomatic state rather than an early asymptomatic hyperparathyroid state. On the other hand, they also observed that a considerable number of patients with the longest follow-up (8 years) did not show evidence of disease progression and may never develop the more typical hypercalcemic phenotype of the disease.

Another observational study by Tordjam et al evaluated 32 individuals with NCHPT. ${ }^{17}$ Several patients had vitamin D deficiency and significant hypercalciuria, but PTH levels remained high after these issues were addressed. Moreover, in 17 patients, the diagnosis of hyperparathyroidism was made after the finding of decreased bone mass during routine bone densitometry and 3 patients had nephrolithiasis at baseline evaluation. Twelve of the normocalcemic

TABLE 1. Clinical presentation and evolution over time of patients with normocalcemic hyperparathyroidism

\begin{tabular}{|c|c|c|c|c|c|c|}
\hline Author & Year & $\begin{array}{c}\text { Total } \\
\text { patients }\end{array}$ & $\begin{array}{l}\text { Median } \\
\text { follow-up } \\
\text { (years) }\end{array}$ & $\begin{array}{l}\text { 25-OHvitD } \\
\text { measurement }\end{array}$ & $\begin{array}{l}\text { Patients characteristics at } \\
\text { diagnosis }\end{array}$ & Disease progression at follow-up \\
\hline Silverberg $^{1}$ & 2003 & 22 & 1 & Yes & $\begin{array}{l}10(45.5 \%) \text { osteoporosis, } \\
1(4.5 \%) \text { fragility fracture, } \\
3(14 \%) \text { kidney stone }\end{array}$ & $\begin{array}{l}3(14 \%) \text { developed hypercalcemia. } \\
\text { Others features were not evaluated }\end{array}$ \\
\hline Tordjam $^{17}$ & 2004 & 32 & 4 & Yes & $\begin{array}{l}12(46 \%) \text { osteoporosis at } \\
\text { lumbar spine and } \\
9(36 \%) \text { at hip, } \\
3(9 \%) \text { nephrolitiasis }\end{array}$ & $\begin{array}{l}12 \text { underwent surgery. } 20 \text { who did not } \\
\text { undergo surgery did not develop hypercal- } \\
\text { cemia/hypercalciuria. Other features were } \\
\text { not evaluated }\end{array}$ \\
\hline Lowe $^{18}$ & 2007 & 37 & 3 & Yes & $\begin{array}{l}27(73 \%) \text { low BMD, } \\
21(57 \%) \text { osteoporosis, } \\
4(11 \%) \text { fragility fracture, } \\
5(14 \%) \text { nephrolitiasis }\end{array}$ & $\begin{array}{l}7(19 \%) \text { developed hypercalcemia, } 1(3 \%) \\
\text { kidney stone, } 1(3 \%) \text { fracture, } 2(5 \%) \\
\text { marked hypercalciuria, } 4(11 \%) \text { new osteo- } \\
\text { porosis, } 6(16 \%) \text { had }>10 \% \text { BMD loss }\end{array}$ \\
\hline
\end{tabular}

*BMD: bone mineral density. 
patients underwent surgery, while the remaining 20 patients did not become hypercalcemic over a mean of $4 \mathrm{yr}$ (range, 1-13yr) of follow-up. The reasons for surgery in these normocalcemic patients are not explained by the authors. Unfortunately, no long-term evaluation of substantial skeletal involvement and development of more features of overt hyperparathyroidism were undertaken in this study.

Previous studies have demonstrated that overt primary hyperparathyroidism is associated with an increased frequency of dyslipidemia, hypertension, overweight and impaired glucose tolerance and increased incidence of cardiovascular morbidity and mortality. ${ }^{6,19-21}$ Several population-based studies have confirmed the association between HPT and cardiovascular diseases, where both calcium and PTH were associated directly or indirectly with increased morbidity and mortality. ${ }^{22-24}$ In this regard, information is sparse in relation to NCHPT and only in one of the studies performed so far does some degree of relationship seem to exist. ${ }^{19}$ However, vitamin D concentrations were not assessed in spite of the important role of vitamin D sufficiency for cardiovascular protection..$^{25}$ A recent study also by Tordjam compared the prevalence of cardiovascular risk factors in patients with NCHPT, in those with hypercalcemic HPT as well as in a control group. ${ }^{26}$ In this study, potential causes of secondary hyperparathyroidism were clearly excluded based on rigorous vitamin $\mathrm{D}$ determinations. In contraposition with a previous study, ${ }^{19}$ the authors reported similar rates of cardiovascular risk factors in the three groups, although the rate of cerebrovascular and ischemic heart clinical disease was more prevalent in hypercalcemic patients when compared with normocalcemic patients and the control population. Curiously, arterial stiffness parameters did not differ between the three groups. However, these findings should be interpreted with some caution, as the study size was quite limited and long-term prospective data are needed to confirm these results.

\section{TREATMENT}

Asymptomatic HPT treatment is a controversial entity as it deals with a condition in which "by definition" there is a lack of specific symptoms or signs, at least those traditionally associated with hypercalcemia. The Third Workshop on the Management of
Asymptomatic Primary Hyperparathyroidism in 2009 emphasized the dilemma of whether a surgical or medical intervention is the best strategy and if severity of the "potential attributable" manifestations of disease and age of the patient should be taken into consideration in the decision-making process. ${ }^{2}$ From the First Workshop on the Management of Asymptomatic Primary Hyperparathyroidism in 1990 to the present time, surgical indications have been evolving towards an earlier parathyroid surgery. However, a clear statement on treatment recommendations of NCHPT was not issued. In fact, the 2009 Workshop concluded that there are insufficient data on the natural history of NCHPT that could be useful for predicting those patients at risk of evolution to a more active form of the disease. Thus, as not all patients have a progression to a hypercalcemic hyperparathyroidism state, and no markers of disease progression are yet available, the decision regarding aggressive treatment versus follow-up must be individualized. Further research into NCHPT natural history is warranted.

It is our impression that most clinicians choose to follow up patients with NCHPT. However, since a number of these patients may progress to a hypercalcemic state or develop the classic conditions requiring surgical intervention such as fractures and kidney stones, it is important to guarantee vigorous follow-up for all these patients. Provision must thus be made that this is both possible and affordable by the patient and by the healthcare budget, with no recommendations on the length of time for followup being applicable in these cases. Without doubt, surgery is an attractive option, as it gives or may give a definitive solution for some patients, mostly those for whom "just follow-up" may be harmful or is simply not recommended for several reasons, e.g. potential lack of compliance with follow-up. However, it is also recognized that medical management (selected estrogen receptor modulators, biphosphonates and calcimimetics) may also be a reasonable option in a substantial number of individuals who do not meet surgical indications or are unable or unwilling to proceed with parathyroidectomy. The aim of such a medical treatment would obviously be to safeguard against NCHPT-mediated tissue damage, as PTH normal levels are not possible to be achieved following these types of treatment. Due to the fact that scientific evidence is scarce, the personalized approach is 
required to balance the benefits and disadvantages of every recommendation, with focus on the particular circumstances of a given patient.

\section{SURGICAL TREATMENT}

Current guidelines for surgical management of asymptomatic $\mathrm{HPT}^{27}$ do not make specific comments regarding NCHPT patients. In fact, postoperative PTH levels may be the only way to determine a successful parathyroidectomy in a NCHPT patient in whom a surgical treatment has been proposed. What is more, studies observe that surgical failure due to multiple glandular disease and negative localization techniques results are more common in NCHPT as compared to hypercalcemic HPT. ${ }^{3}$ Thus, as multiglandular disease and operative failure seem to be more frequent in NCHPT and a more extensive bilateral neck exploration and multiple glandular excision are required, this higher risk for surgical morbidity presupposes that the decision to propose such a step would be convincingly supported and clearly explained to the patient. ${ }^{28}$

As in classical HPT, surgical experience is the main variable determining the like hood of surgical complications during parathyroidectomy. Therefore, the number of cases operated on per year by a given surgeon is the most important predictor of the clinical outcome in parathyroid surgery, and this is particularly true for NCHPT in which the gland to be removed may not be very large and, as a consequence, much more difficult to identify. The type of surgical approach and the use of intra- or peri-operative supportive procedures is highly institution-specific and should be based on the expertise and resource availability of the surgeon and the institution. In this regard, the intra-operative determination of PTH may be very helpful for confirmation of surgical success. Preoperative localization techniques of an enlarged parathyroid gland (sestamibi scan, CT scan, MRI, ultrasonography, etc.) in patients with NCHPT may be more difficult to interpret, as the target gland could be less large than in classic hypercalcemic HPT and, as mentioned, the likelihood of multiple-gland disease is not negligible. 99mTC-Sestamibi is probably the most frequently used and sensitive probe for parathyroid localization. Its main advantage is its ability to detect ectopic parathyroid glands while also providing functional information. Neck ultrasound plays a principal role in thyroid nodule and parathyroid gland enlargement evaluation but it is highly dependent on the experience of the radiologist performing the study; ${ }^{29}$ its use in the immediate preoperative scenario may also be of help for the surgeon. Combinations of both non-invasive techniques as complementary methods are highly recommended because of their safety and sensitivity. However, the predictive value of both localization techniques tended to be lower in patients with NCHPT. Some studies have demonstrated a lower sensitivity in NCHPT, especially in multigland disease, in comparison with hypercalcemic HPT. The median gland weight in a NCHPT cohort may be lower than hypercalcemic HPT and this probably explains the decreased sensitivity of preoperative imaging. Given the relatively low sensitivity of each imaging study individually, the use of both imaging modalities together will increase the overall sensitivity of preoperative localization and may be recommended. ${ }^{30}$

In special circumstances other imaging techniques could be of interest (CT scan, MRI, PET scan), and in extreme cases invasive procedures, such as parathyroid fine needle aspiration, arteriography and/or selective venous sampling for $\mathrm{PTH}$, may be considered. However, all these procedures are expensive and time-consuming, entailing minor but not negligible risks, and should be proposed in a very limited way for guiding minimally invasive surgery where localization of the affected parathyroid gland is essential, or in those cases without information using conventional non-invasive techniques. ${ }^{27}$ Particularly in NCHPT, the difficulties involved in proceeding to minimally invasive surgery and the high risk of these localization techniques restrain their use in almost all situations.

On the other hand, a new clinical entity, postoperative NCHPT, has recently been described following surgery, this being a not uncommon situation in patients after an apparently successful parathyroidectomy for hypercalcemic HPT. At present, no definitive explanation for this postoperative phenomenon is available. Although most studies report a percentage of recurrent disease of approximately 5\%-10\% following a successful surgery, this new clinical entity must be seen as the first phase of the recurrence of hypercalcemic HPT or some kind of vitamin D in- 
sufficiency or resistance secondary to drop of PTH level. ${ }^{31}$ In fact, some studies have shown that oral vitamin D supplementation reduces the incidence of eucalcemic PTH elevation after surgery for primary hyperparathyroidism..$^{32}$ In summary, there is no established definition criteria of surgical cure for NCHPT patients, which to date remain based on the full normalization of circulating PTH in the context of a normal vitamin D status.

\section{MEDICAL TREATMENT}

Medical treatment could be an interesting approach for those patients with NCHPT who do not meet surgical indications or are unable or unwilling to proceed with parathyroidectomy under a personalized approach. In this case, the aim of such a medical treatment would be to protect possible NCHPT-induced complications and not to attain normalization of PTH levels. Accordingly, at present medical treatment is limited to those individuals in whom disease progression or bone alterations are suspected. Further investigation is required in this field.

Selective estrogen receptor modulators and bisphosphonates could be of interest in NCHPT treatment because of potential BMD (bone mineral density) increase and fracture risk reduction. Unfortunately, none of these treatments have been evaluated in clinical assays, neither in hyperparathyroidism nor in NCHPT. ${ }^{33}$ Only alendronate treatment was shown to improve BMD in the lumbar spine of patients with primary hyperparthyroidism without changes in calcium levels to a degree comparable to that achieved in eucalcemic populations. ${ }^{34}$ Furthermore, none of the treatments evaluated to date have included data on fracture outcome.

Calcimimetics, which comprise a new pharmacologic class, modulate the calcium sensing receptor (CaSR), increasing the sensitivity of this receptor to the extracellular calcium. Cinacalcet has been shown to be effective in reducing or normalizing serum calcium levels in several groups of patients with primary hyperparathyroidism with slight reduction of PTH levels and no effects on bone mineral density. ${ }^{35-36}$ Until now there are no data as to whether long-term treatment with cinacalcet can prevent the complications/progression of NCHPT. On this account, and also because of the high cost of this drug, its use should be avoided.

\section{CONCLUSION}

NCHPT may have a heterogeneous phenotypic presentation and an unpredictable evolution. In some patients there is a progression of the disease and in others there is not, and until now there is no way to identify both cohorts and prediction of progression is not currently feasible. It thus far remains unclear whether it constitutes the initial phase or/and an indolent form of classic hyperparathyroidism. Data suggest that it may not be the early stage of mild asymptomatic hyperparathyroidism with any health consequence but the earliest and/or a mild form of symptomatic hyperparathyroidism, suggesting that this entity may not be as indolent as previously thought. Very little is known about the actual prevalence, pathophysiology and natural history of NCHPT. Moreover, there are as yet no guidelines for the management of NCHPT and it is unknown if therapeutic intervention aimed to normalize PTH may have any true benefits in the short and long term in these patients. It is therefore imperative to conduct studies in order to remedy this extensive lack of information and, most importantly, to seek to identify markers of disease progression crucial for prediction of those cases developing an active disease.

No grant or fellowship support.

\section{REFERENCES}

1. Silverbeng SJ, Bilezikian JP, 2003 "Incipient" primary hyperparathyroidism: a "forme fruste" of an old disease. J Clin Endocrinol Metab 88: 5348-5352.

2. Bilezikian JP, Khan A, Potts JT, 2009 Guidelines for the management of asymptomatic primary hyperparathyroidism: summary statement from the third international workshop. J Clin Endocrinol Metab 94: 335-339.

3. Carneiro-Pla D, Solorzano C, 2012 A summary of the new phenomenon of normocalcemic hyperparathyroidism and appropriate management. Curr Opin Oncol 24: 42-45.

4. Wills MR, Pak CY, Hammond WG, Bartter FC, 1967 Normocalcemic primary hyperparathyroidism. Am J Med 47: 384-391.

5. Monchik JM, Gorgun E, 2004 Normocalcemic hyperparathyroidism in patients with osteoporosis. Surgery 136:1242-1246.

6. Silverberg SJ, Lewiecki EM, Mosekilde L, Peacock M, Rubin MR, 2009 Presentation of asymptomatic primary hyperparathyroidism: proceedings of the third international workshop. J Clin Endocrinol Metab 94: 351-365.

7. Bilezikian JP, Silverbeng SJ, 2010 Normocalcemic pri- 
may hyperparathyroidism. Arq Bras Endocrinol Metab 54: 106-109.

8. Eastell R, Arnold A, Brandi ML, et al, 2009 Diagnosis of Asymptomatic Primary Hyperparathyroidism: Proceedings of the Third International Workshop. J Clin Endocrinol Metab 94: 340-350.

9. Souberbielle JC, Cormier C, Kindermans C, et al, 2001 Vitamin D status and redefining serum parathyroid hormone reference range in the elderly. J Clin Endocrinol Metab 86: 3086-3090.

10. Looker AC, Pfeiffer CM, Lacher, DA, et al, 2008 Serum 25-hydroxyvitamin D status of the US population: 1988-1994 compared with 2000-2004. Am J Clin Nutr 88: 1519-1527.

11. Chapuy MC, Arlot ME, Duboeuf F, et al, 1992 Vitamin D3 and calcium to prevent hip fractures in the elderly women. N Engl J Med 327: 1637-1642.

12. Chapuy MC, Pamphile R, Paris E, et al, 2002 Combined calcium and vitaminD3 supplementation in elderly women: confirmation of reversal of secondary hyperparathyroidism and hip fracture risk: the Decalyos II study. Osteoporos Int 13: 257-264.

13. Dawson-Hughes B, Harris SS, Krall EA, Dallal GE, 1997 Effect of calcium and vitamin supplementation on bone density in men and women 65 years of age or older. N Engl J Med 337: 670-676.

14. Harvey A, Hu M, Gupta M, et al, 2011 A New, Vitamin D- Based, Multidimensional Nomogram for the Diagnosis of Primary Hyperparathyroidism. Endocr Pract 8: 124-131.

15. Lundgren E, Hagstrom EG, Lundin J, et al, 2002 Primary hyperparathyroidism revisited in menopausal women with serum calcium in the upper normal range at population based screening 8 yrs ago. World J Surg 226: 931-936.

16. Maruani G, Hertig A, Paillard M, Houillier P, 2003 Normocalcemic primary hyperparathyroidism: evidence for a generalized target-tissue resistance to parathyroid hormone. J Clin Endocrinol Metab 88: 4641-4648.

17. Tordjman KM, Greenman Y, Osher E, et al, 2004 Characterization of normocalcemic primary hyperparathyroidism. Am J Med 117: 861-863.

18. Lowe H, McMahon DJ, Rubin MR, et al, 2007 Normocalcemic primary hyperparathyroidism: further characterization of a new clinical phenotype. J Clin Endocrinol Metab 92: 3001-3005.

19. Hagström E, Lundgren E, Rastad J, Hellm P, 2006 Metabolic abnormalities in patients with normocalcemia hyperparathyroidism detected at a population-based screening. Eur J Endocrinol 155: 33-39.

20. Hedbäck G, Odén A, 1998 Death risk factor analysis in primary hyperparathyroidism. Eur J Clin Invest 28: 1011-1018.

21. Valdermarsson S, Lindblom P, Bergenfelz A, 1998 Metabolic abnormalities related to cardiovascular risk in primary hyperparathyroidism: effects of surgical treatment. J Interm Med 244: 241-249.
22. Hedbäck G, Odén A, 1998 Increased risk of death from primary hyperparathyroidism- an update. Eur J Clin Invest 28: 271-276.

23. Jorde R, Sundsfjord J, Fizgerald P, Bonaa HK, 1999 Serum calcium and cardiovascular risk factors and diseases: the Tromsö study. Hypertensión 34: 484-490.

24. Jorde R, Bonaa KH, Sundsfjord J, 1999 Population based study on serum ionised calcium, serum parathyroid hormone, and blood pressure: the Tromsö study. Eur J Endocrinol 141: 350-357.

25. Adams JS, Hewison M, 2010 Update in vitamin D. J Clin Endocrinol Metab 95: 471-478.

26. Tordjam KM, Yaron M, Izkhakov E, et al, 2010 Cardiovascular risk factors and arterial rigidity in asymptomatic normocalcemic and hypercalcemic primary hyperparathyroidism. Eur J Endocrinol 162: 925-933.

27. Udelsman R, Pasieka JL, Sturgeon C,Young JEM, Clark OH, 2009 Surgery for Asymptomatic Primary Hyperparathyroidism: Proceedings of the Third International Workshop J Clin Endocrinol Metab 94: 366-372.

28. Carneiro-Pla DM, Romaguera R, Nadji M, Lew JI, Solorzano CC, Irvin GL 3rd, 2007 Does histopathology predict parathyroid hypersecretion and influence correctly the extent of parathyroidectomy in patients with sporadic primary hyperparathyroidism? Surgery 142: 930-935.

29. Soto GD, Halperin I, Squarcia M, Lomeña F, Domingo MP, 2010 Update in thyroid imaging. The expanding world of thyroid imaging and its translation to clinical practice. Hormones (Athens) 9: 287-298.

30. Wade TJ, Yen TW, Amin AL, Wang TS, 2012 Surgical management of normocalcemic primary hyperparathyroidism. World J Surg 36: 761-766.

31. Goldfarb M, Gondek S, Irvin GL 3rd, Lew JI, 2011 Normocalcemic parathormone elevation after successful parathyroidectomy: long-term analysis of parathormone variations over 10 years. Surgery 150: 1076-1084.

32. Beyer TD, Solorzano CC, Prinz RA, Babu A, Nilubol N, Patel S, 2007 Oral vitamin D supplementation reduces the incidence of eucalcemic PTH elevation after surgery for primary hyperparathyroidism. Surgery 141: 777-783.

33. Khan AA, Bilezikian JP, Potts JJ, 2009 Asymptomatic primary hyperparathyroidism: a Commentary on the revised guidelines. Endocr pract 15: 494-498.

34. Khan AA, Bilezikian JP, Kung AW, 2004 Aleudronate in primary hyperparathyroidism: a doubleblind, randomized, placebo-controlled trial. J Clin Endocrinol Metab 89: 3319-3325.

35. Marcocci C, Cetani F, 2012 Update on the use of cinacalcet in the management of primary hyperparathyroidism. Endocrinol Invest 35: 90-95.

36. Peacock M, Bilezikian JP, Klassen PS, Guo MD, Turner SA, Shoback DM, 2005 Cinacalcethydrochloride maintains long-term normocalcemia in patients with primary hyperparathyroidism. J Clin Endocrinol Metab 90: 135-141. 